\title{
APPLYING SIP B2BUA FOR MOBILE WIMAX NETWORK ARCHITECTURE
}

\author{
Yen-Wen Chen, Meng-Hsien Lin, and Hong-Jang Gu \\ Department of Communication Engineering \\ National Central University \\ 320 No. 300, Jhong-Da Road, Jhong Li City, Taiwan ROC \\ ywchendce.ncu.edu.tw
}

\begin{abstract}
This paper proposes a novel mobility integration method and heuristic algorithm in WiMax network. The objective of the method focuses on cooperation of the ASN anchored mobility and SIP mobility and uses the B2BUA concept to improve the handoff performance as well as to reduce the handoff dropping rate. As the B2BUA acts as a proxy to handle the link layer and application layer handoffs, the handoff decision and policy can be enforced in a heuristic manner. Experimental simulations were performed and the results show that the proposed scheme can effectively utilize the traffic links to achieve the aforementioned objective.
\end{abstract}

\section{KEYWORDS}

WiMax, Session Initiation Protocol, Mobile Network, ASN Anchored Mobility, SIP Mobility,B2BUA

\section{INTRODUCTION}

As the networks gradually evolved from the wired environment (Ethernet IEEE802.3) to today's wireless one (Wireless IEEE802.11), the restrictions posed by the wired network have become history. In recent years, more and more mobile devices, such as smart phones, tablet computers, and personal digital assistants (PDA), have been extensively adopted. New applications, such as Voice over IP (VoIP), Video on Demand (VoD), real time streaming, online games, and Social Network Services (SNS), continue to be developed to meet emerging service demands. These applications, when being executed in mobile environment, require seamless handoff to achieve high quality service in real wireless networks.

The Worldwide Interoperability for Microwave Access (WiMax) [1, 2] technology has been proposed to support broadband services in mobile environment. In order to provide complete end-to-end service scenario, the network is divided into access service network (ASN) and connectivity service network (CSN) with several control modules inside them to deal with the required signaling functions. The end-to-end architecture provides the ASN anchored mobility and the CSN anchored mobility for efficient handoff. The ASN anchored mobility uses tunnel to keep the transmission path prior to the anchor point unchanged, and therefore, has smooth transmission performance and lower handoff latency. However, the path between mobile node $(\mathrm{MN})$ and correspondent node $(\mathrm{CN})$ may not be the optimal arrangement and may waste network resources, with triangle route problem as an example. On the other hand, CSN anchor mobility is more efficient from the resource utilization point of view, however, the end-to-end path needs to be re-routed $[3,4]$ and hence causes longer handoff latency.

The WiMax Forum Network Working Group have proposed the client based and proxy based Mobile IPv4 (MIPv4) and Mobile IPv6 (MIPv6) to cooperate with existing link layer mobility. 
However, the mobile node must be equipped with mobile IP capability to retain the connectivity at network layer, a condition not to be assumed in real environment. Since the network layer function is always tightly coupled with the operating system kernel in most mobile devices, it is not readily accessible to most people. Therefore, providing handoff capability at application layer may be an alternative way to achieve the mobility without changing device network protocol. The Session Initiation Protocol (SIP) [5] is basically a user based mobility approach. The user identification, or alias, is the key to maintain the service connectivity while the IP mobility is not essential. By registering the current location (e.g. IP address) of the user identification at the SIP server, other users can easily query user location at the SIP server and communicate with the user in a peer to peer manner. Furthermore, the re-invite procedure allows the user to inform the peer users of location change during movement. However, as SIP is an application layer protocol, the SIP based mobility cannot improve the performance of latency and packet loss suffered from connection handoff [6, 7]. By using the soft handoff approach, which establishes the new connection before breaking the old connection, it is helpful for the improvement of the latency.

In this paper, we adopt the hybrid ASN anchored and SIP mobility as the basic concepts for soft handoff and propose the back-to-back user agent (B2BUA) at ASN node, referred as B2BUA-ASN node, as a proxy role to send invite and re-invite messages to $\mathrm{CN}$ to achieve handoff efficiently. A B2BUA is a logical network entity in SIP environment, which can receive the UAC message and use the Call Control Logic to transmit the other UA. The B2BUA can initiate the new call and modify the SIP message. Thus, the mobile node can be mobile IP unaware in the B2BUA-ASN node. In addition, as the ASN node plays the role of anchor point for connection handoff $[8,9,10]$, the integration of B2BUA at ASN node can decide, based on the traffic condition, whether the handoff connection is ASN anchored or not in a heuristic manner, so that the network resource can be more effectively utilized. This paper studies the operational scenario of B2BUA-ASN based on the existing WiMax handoff procedure.

\section{OVERVIEW OF Mobility MaNagement ANd RELATEd WORKS}

The mobility management is one of the most important issues in the wireless network environment, since it covers several protocol layers and network interfaces. The handoff performance is measured by several criteria. Generally, the latency and packet loss during handoff are the most important performance indexes that are frequently analyzed. It is noted that the network loading, including the network node and the link bandwidth, should be considered in handoff process. The WiMax forum defines a system wide architecture with several reference interfaces. From the link layer point of view, several possible mobility scenarios exist at each reference interface as shown in Figure 1.

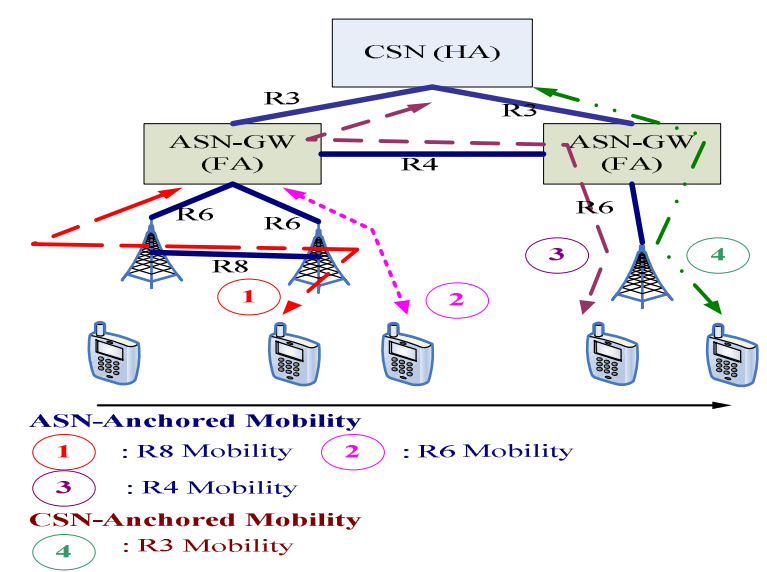

Fig. 1. WiMax Mobility Scenarios 
In addition to the link layer handoff, the WiMAX end-to-end architecture documents [10, 11] also defined the network layer mobility. Furthermore, the application layer mobility is also widely applied at each end devices. These three mobility architectures are described as follows:

- Link Layer Mobility (CSN/ASN anchored)

In the WiMAX network architecture, two scopes of mobility have been proposed: one is ASN anchored mobility and the other is CSN anchored mobility. ASN anchored mobility refers to a set of procedures associated with the movement between two BSs. The target BS can be in the same ASN or in a different one. The anchor ASN remains identical during ASN anchored mobility. ASN anchored mobility takes place when the MS moves to a neighboring BS that connects to a different ASN GW, which may be within the serving ASN or not. If the target BS belongs to a different ASN, the target ASN-GW will establish a R4 data path to the anchor ASN. This way the MS can avoid data loss and the QoS can be guaranteed as well. During the ASN anchored handoff procedure, the MS does not change its CoA. For ASN anchored mobility, traffic to the MS comes from the anchor ASN. CSN anchored mobility involves anchor ASN relocation, which means anchor ASN is changed after CSN anchored mobility. After CSN anchored mobility execution, MS will update its CoA to the target ASN. The CoA renewal process uses Client MIPv4 (CMIPv4) or Client MIPv6 (CMIPv6) or Proxy MIPv4 (PMIPv4) protocol. And the network will trigger the data path function relocate so that the CSN will directly transmit the data to the target ASN. The target ASN-GW is now becoming the anchor ASN-GW and serving ASN-GW to the MS. The procedure is known as R3 re-anchoring, because the traffic to MS comes from the new anchor point, which is a new R3 link. When CSN anchored mobility is executed, it is involved in CoA update. ASN anchored mobility prior to the CSN anchored mobility minimizes the handoff delay and packet loss.

\section{- Network layer (Client MIP, Proxy MIP)}

CMIP is used in the standard (RFC-3344) of the Mobile IP technology. The MS must support Mobile IP functionality. When MS detected that they have moved, they will use the Mobile IP technology to update the network status. Proxy Mobile IP (PMIP) provides mobility support without changing the network layer protocol of these devices. It is used in WiMAX, 3GPP and 3GPP2 architectures. PMIP network is the Network infrastructure that helps MN achieve the purpose of MIP. The MN doesn't need to have the MIP ability, but simply follows the original usage of the network (such as DHCP agreement), PMIP include a MAG (Mobility Access Gateway) in the Mobile IP architecture which interacts with the HA and the LMA (Local Mobility Anchor) on behalf of the Mobile Node. MAG is an element on an access router that manages the mobility for a MS. LMA is the home agent for the MS in PMIP.

\section{- Application Layer (SIP)}

SIP is an application-layer protocol, which is used in establishing and tearing down voice or video calls. SIP has high flexibility and high extension advantages. SIP supporting VoIP mobility becomes increasingly more important and supports four mobile mechanisms:

1. Terminal mobility: Allows terminal devices to move to different domain or subnet. To ensure that the packets still get delivered correctly, or that the device maintains the current participants of the session.

2. Session mobility: Allows the user to switch to a different terminal device during the SIP session, while still allowing the user to maintain call quality and to keep the connection. 
International Journal of Computer Networks \& Communications (IJCNC) Vol.3, No.5, Sep 2011

3. Personal mobility: Allows different terminal devices to use the same SIP address or a terminal device to use multiple SIP addresses at the same time. For example: a SIP user can use the PC and mobile phone to register the same SIP URI at, and the user only have to remember the URI.

4. Service mobility: Allows the user to switch to a different network, to different network devices, or to different Internet service and original services can still be retained.

The SIP mobility presented in this paper is Terminal Mobility. As mentioned before, SIP (Terminal) Mobility allows terminal device to move to different domain or subnet. So the user can use the re-invite method to ensure that the packets still get delivered correctly, or that the device maintains the current participants of the session.

Although, PMIP is the network-based approach that helps MS achieve the purpose of IP mobility and the MS doesn't need to have the MIP ability, still not all network infrastructures support PMIP, as it is decided by the network operators. When using the VoIP application, we must use the SIP to initialize communication. As the SIP mobility is an application layer protocol, one of its advantages is that it can be readily deployed on the network, as the network protocol of MS and network do not require any further modification. SIP register approach is similar to MIP approach, although the MS does not need to fix HoA (Home Address) in SIP register approach.

Consequently, we propose an architecture that combines the ASN, CSN anchor and SIP mobility and also builds the B2BUA component into the current WiMAX architecture to overcome or to improve existing drawbacks.

\section{The Proposed Hybrid ASN ANChORED/SIP MobILITY MANAGEMENT SCHEMES}

This paper proposes a hybrid architecture, which dynamically adopts SIP mobility through B2BUA and anchor mobility at the ASN component, to improve the handoff performance. When the MS is powered on, it first accesses WiMAX network and completes the ND\&S (Network Discovery and Selection). Then the MS performs the SBC (Station Basic Capability) negotiation and authentication and notifies the core network of MS support capability of SIP mobility. We add two bits of the optional file on extended capability [9] to specify whether the MS supports the SIP mobility and network assistant SIP mobility or not. After completing the access authentication, the MS carries out the connection setup process. Once the MS gets the IP address, it will register to the SIP server. If the MS is equipped with CMIPv4 capability, it can individually perform network layer mobility after the SIP connection is established, as shown in Figure 2. 
International Journal of Computer Networks \& Communications (IJCNC) Vol.3, No.5, Sep 2011

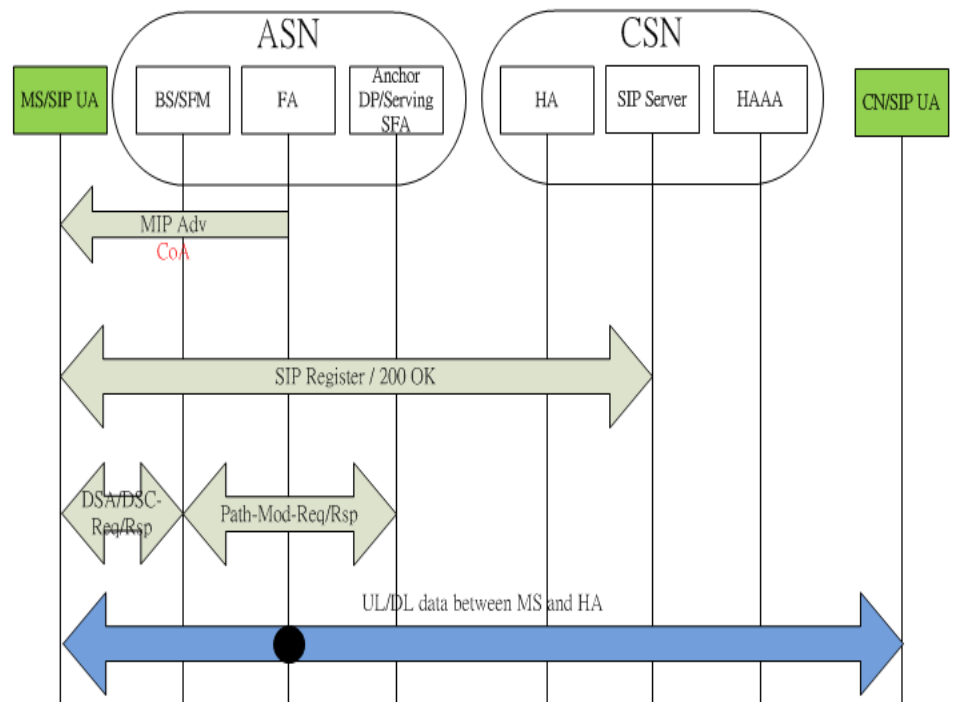

Fig. 2. Connection setup - SIP mobility (CMIPv4)

Although SIP solves the MIP routing optimization and HA bottleneck problem, it will cause more delay latency in certain conditions, as SIP is an application layer solution. , For example, when MS gets new IP address, it transmits re-invite message to the CN. The re-invite message is delivered via R1, R6, R3 interface and Internet network to the $\mathrm{CN}$ and this causes some delay latency. During the period of transmitting re-invite message, there may also be package loss, because the package form the $\mathrm{CN}$ to the MS will be transmitted to the old ASN. Therefore before carrying out SIP mobility, we employ the ASN anchored mobility to build Data Path of the serving and target ASN. It can pass the package to old ASN through R4 Data Path tunnel to new ASN, and that can reduce the SIP re-invite message impact. We illustrate the scenario as in the Figure 3.

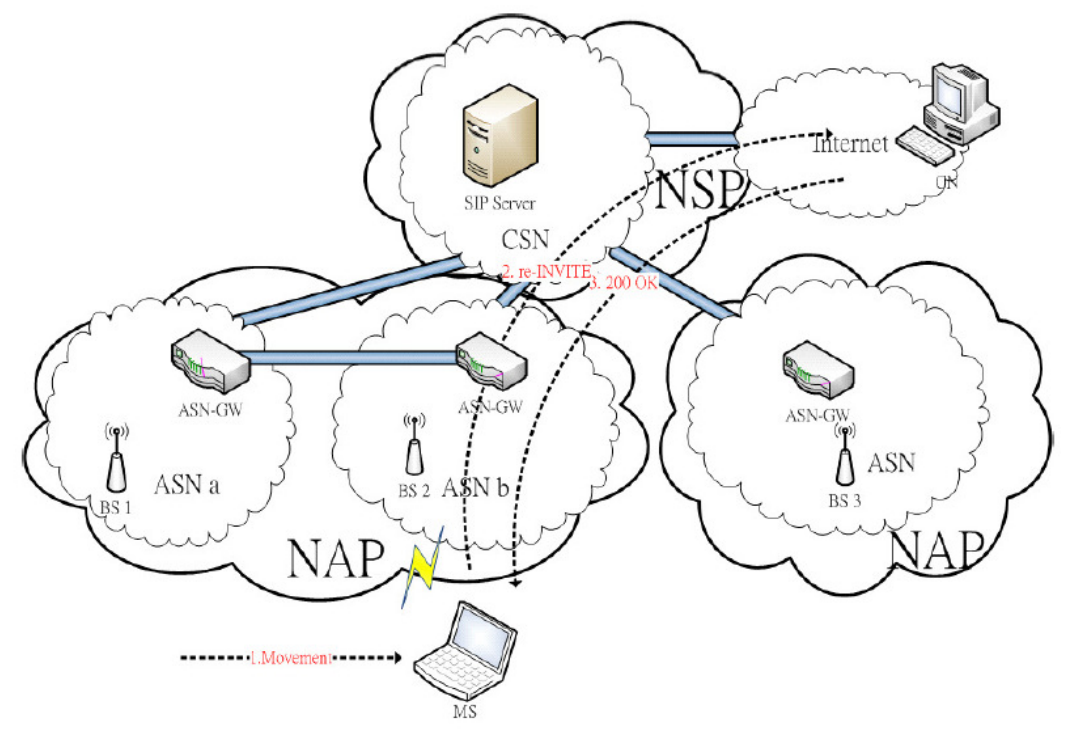

Figure 3 The Architecture of ASN Anchored Mobility combines SIP Mobility 
When the MS moves from ASN a to ASN b, the core network processes the ASN anchored mobility by setting the Data Path of ASN-GW between ASN a and ASN b. Then the MS get new CoA and the SIP UA of MS will transmit re-invite message to SIP UA of CN. The packet deliver from ASN a to ASN b through the R4 Data Path tunnel. After the CN receives the reinvite message, the CN transmits the new data to new CoA and responds " 200 OK" message to the MS. The ANS-GW of ANS b triggers the action of destroying the R4 Data Path when it receives the "200 OK" message. The ASN anchored mobility combining SIP mobility flow chart based on MIPv4 is shown in Figure 4.

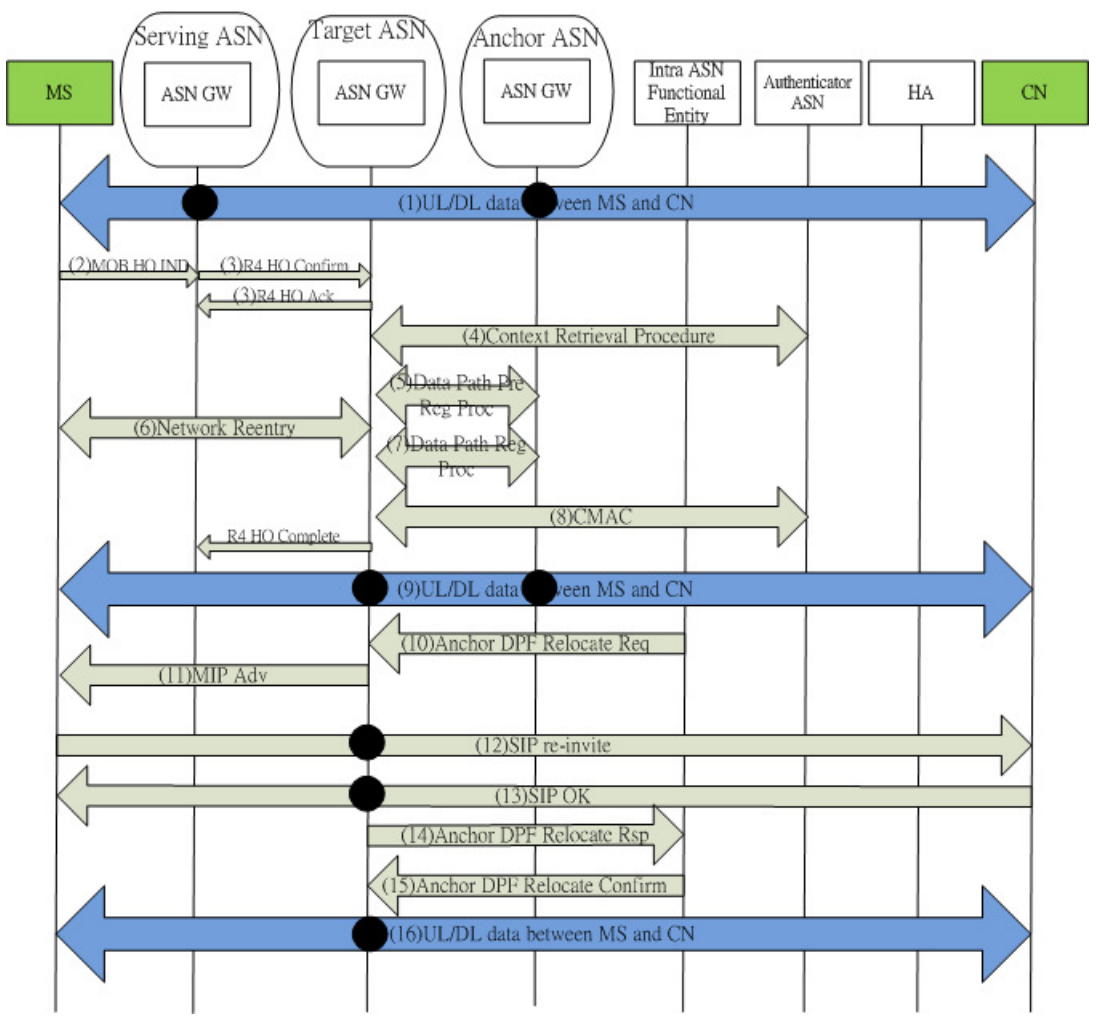

Fig. 4. The ASN Anchored mobility combines SIP mobility flow chart (CMIPv4)

A detailed flow is described as follows:

- (1) Before the MS moves, it transmits and receives data through serving ASN and anchor ASN;

- (2) (8) ASN anchored mobility process ;

- (9)When completing the ASN anchored mobility, the MS transmits and receives data through target ASN and anchor ASN ;

- (10) (11) the Intra-ASN Functional Entity triggers Anchor_DPF_Relocate_Req Message and passes it to target ASN. The foreign agent of target ASN broadcasts Mobile IP Advertisement to the MS. Then the MS can get the new CoA.

- (12) (15) The MS transmits re-invite message to the $\mathrm{CN}$, then the $\mathrm{CN}$ responds with the 200 OK message through target ASN-GW. The target ASN-GW sends Anchor DPF 
Relocate Rsp message to Intra-ASN functional entity. The process will destroy the R4Data Path.

- (16) When the MS completes SIP mobility, MS transmits and receives data through target ASN directly.

Although doing the ASN anchored mobility first then the SIP mobility can reduce time delay of the re-invite message and packet loss, the MS must transmit the re-invite message through R1, hence the re-invite message will be delayed if the BS is overloaded. Even though we can modify the QoS schedule to give the SIP message higher priority, we cannot avoid the packet loss or damage due to wireless signal interference. For this reason, when the MS moves to the new ASN, the core network helps the MS to transmit re-invite message to update CoA. This can shorten the time for sending re-invite message.

In WiMAX Stage $2 / 3$ definition, the core network can also support proxy mobile IP (PMIP) to assist the MS that has no mobile IP (MIP) capability. And both MSs of a SIP connection need MIP functions within the mobile environment if they want to perform handoff at network layer. Alternatively, the SIP mobility provides the application layer mobility when the MS has no MIP capability. We propose to support the B2BUA function at the ASN component as shown in Figure 5. The B2BUA is a logical entity; it can receive the UAC message and use the Call Control Logic to transmit the other UA. The B2BUA acts as a UA point of the correspondent node as well as a UA end point of the MS and can initiate new calls and modify SIP messages. So we put the B2BUA in the ASN as shown in Figure 3.8. The B2BUA handles the MS register message and registers MS to the SIP Server for MS, records the invite message of MS and stores the MAC address and invite message in the database.

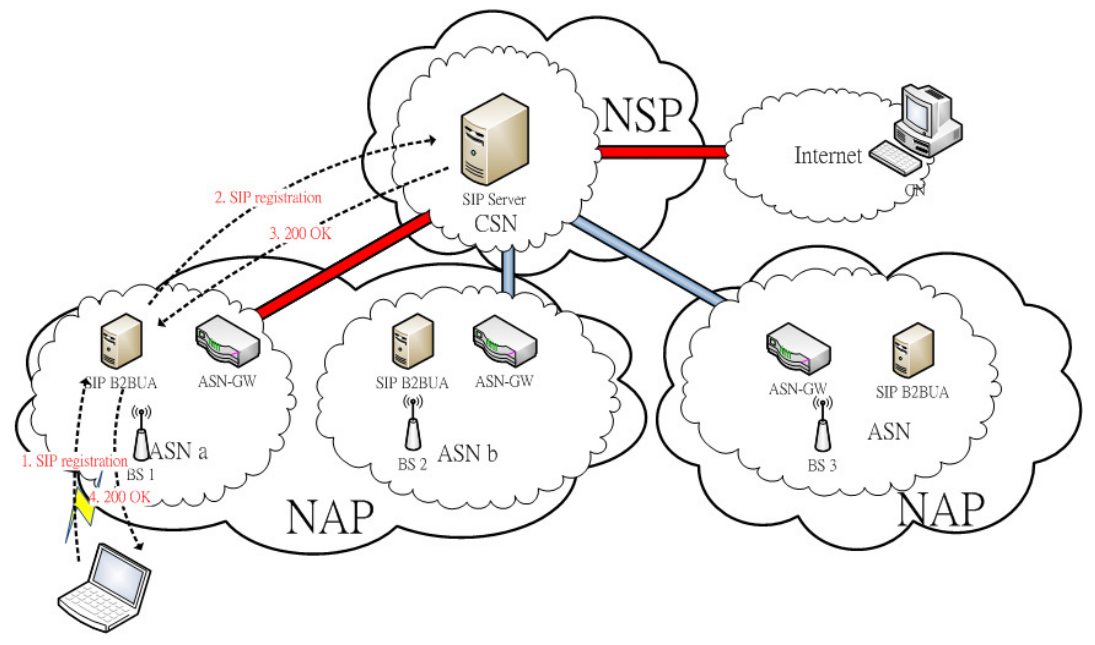

Fig. 5. Network assistance SIP mobility in WiMAX network with B2BUA

Without network layer capability, the MS will initiate SIP re-invite procedure when it moves to the other routing domain and receives a new IP address through the DHCP server. The MS is only notified of the new IP address, however, the network layer will not report the CoA to its correspondent node due to lack of MIP capability. Instead, the MS will send the re-invite message to its peer, i.e. the B2BUA, for the new IP address. As the layer 2 handoff should occur when the routing domain changes, therefore the cross layer handoff decision will be considered at the ASN GW. Upon the receipt of the re-invite message, the ASN GW can decide to employ either ASN anchored mobility or SIP mobility. If MS is in ASN anchored mobility, it will not 
International Journal of Computer Networks \& Communications (IJCNC) Vol.3, No.5, Sep 2011

need to resend the re-invite message to the correspondent SIP client. Otherwise, it will resend the re-invite message to the correspondent SIP client. If the ASN anchored mobility is taken, there will be no change at the correspondent SIP client, only the layer 2 handoff will occur between the moving MS and the ASN GW. In this case, the path between the correspondent SIP client and the previous ASN GW stays the same and a tunneling data path will be established at R4 interface (i.e. between the previous ASN GW and the serving ASN GW). On the other hand, if SIP mobility is taken, the B2BUA at the serving ASN GW will send the re-invite message to the correspondent SIP client to report the new IP address. In this case, R3 interface mobility occurs and the tunneling data path is not necessary. Thus, the B2BUA has the flexibility to decide the routing path during handoff. Regardless of DHCP, MIPv4, MIPv6, the MS can get the IP address then register to the B2BUA. And the B2BUA can further help the MS register to the SIP Server and record invite message to provide re-invite message processing. Since during the initial service flow B2BUA can transmit SIP message, the registration of MS can be expedited?.

In the Figure 6 shows the MS moves to different ASN, then B2BUA helps the MS update message to the $\mathrm{CN}$

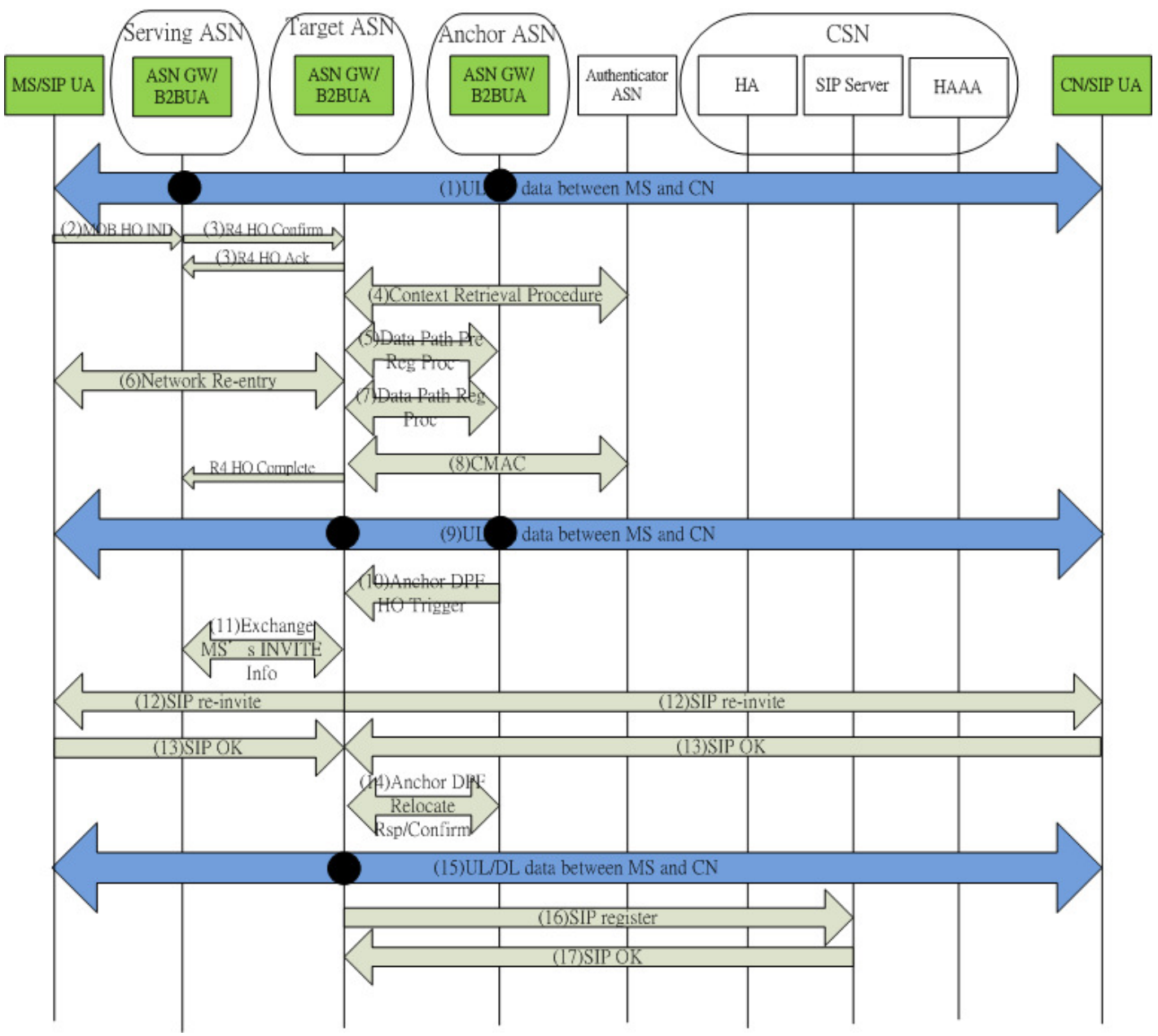

Fig. 6. Network assist SIP Mobility flow chart (DHCP 、CMIP) 
International Journal of Computer Networks \& Communications (IJCNC) Vol.3, No.5, Sep 2011

- (1)Before moving, the MS transmits and receives data through serving ASN and anchor ASN;

- (2) (8) ASN anchored mobility process;

- (9)When completing the ASN anchored mobility, the MS transmits and receives data through target ASN and anchor ASN;

- (10)If anchor ASN decides to process R3 Re-anchor, it will transmit Anchor_DPF_Relocate_Trigger message to target ASN. If the target ASN agrees to make R3 Re-anchor, it will process step (11), else the step can be skipped;

- (11) The target B2BUA (B2BUA of target ASN) triggers serving B2BUA (B2BUA of serving ASN) and transmits the preservative invite message to target B2BUA according to the MAC address of MS. The target B2BUA increases Cseq in SIP header and modify the Contact and (c) in SDP field.

- (12) (14)The target B2BUA transmits the re-INVITE message to the MS and the CN, if they receive it, they will respond with 200 OK message. The target B2BUA uses the message to trigger target ASN-GW for transmitting Anchor DPF Relocate Rsp message to Anchor ASN-GW, and then destroys the R4Data Path.

- (15) After completing SIP mobility, the MS transmits and receives data from target ASN directly.

- (16) (17) the registration of MS s to SIP Server is completed.

The Qos of Stage 2/3 definition is shown in Figure 7. Besides integrating the 802.16d and 802.16e, the QoS also includes BS and ASN-GW (R6), ASN-GW and ASN-GW (R4). After the MS handoff, the network can provide the existing QoS for the MS. As we don't find the Stage 2/3 definition with the QoS of R3 included, we may use RSVP to reserve the resource; however all network nodes need to support the RSVP. Before MS handoff, we use existing WiMAX mobility management and SIP mobility to count each ASN-GW R3 loading and R4 loading. When the R3 is overloaded, we process the ASN anchored mobility; when the R4 is overloaded, we process the SIP mobility. Hence using the approach not only can balance the core network loading, but also guarantee the QoS in the WiMAX architecture.

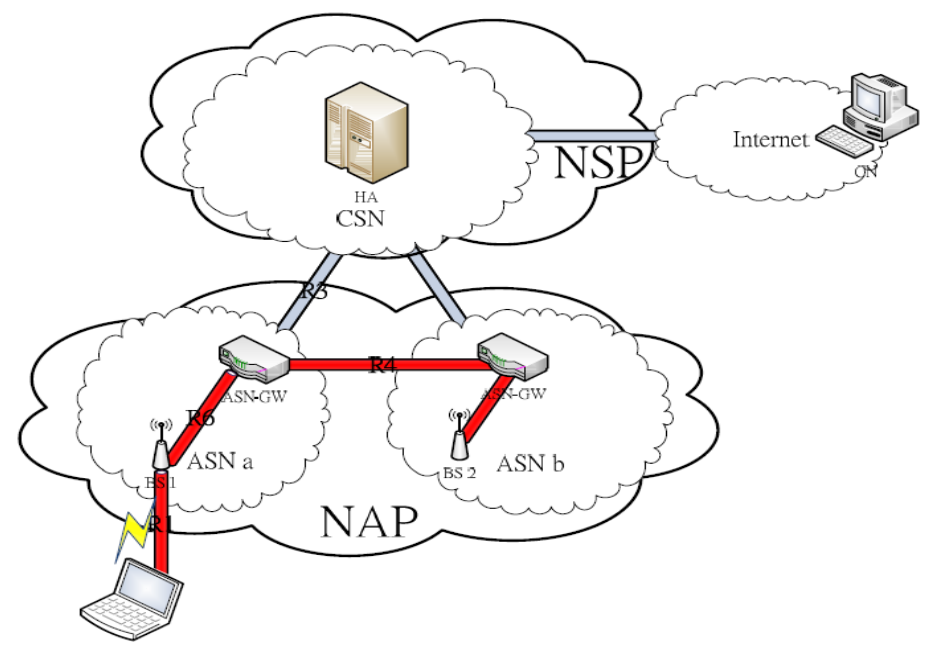

Fig. 7. WiMAX QoS scope 
The handoff decision proposed in WiMax [11, 12] prefers to make the ASN anchored mobility first, and then the CSN anchored mobility. The reason is that ASN anchored mobility doesn't change IP, so it can complete the handoff speedily, thereby reducing the handoff latency. However, the ASN anchored mobility must establish the tunnel between previous (old) ASNGW and the serving (new) ASN-GW, which increases the ASN-GW loading and the packet of tunnel overhead. If the MS moves far away the anchor ASN-GW, it will cause a longer tunneling path and increase the transmission overhead. Regarding CSN anchored mobility (i.e. without tunneling path), its obvious advantage is to reduce the network resource as well as the load of ASN-GW. But the disadvantage is that it takes longer time for the MS to complete handoff. Then the SIP mobility can be regarded as a kind of CSN anchor architecture, which is also named as R3 Re-anchor architecture.

In addition to the proposed B2BUA architecture, a load balance based handoff decision scheme is proposed in this paper. Generally, when considering the downstream traffic, the arrival traffic of ASN GW may be either from R3 interface or the tunneled R4 interface. And as mentioned above, the load of R4 interface increases if the ASN anchored mobility is applied. Therefore, the decision of ASN anchored handoff or SIP mobility should take the R3 and R4 load into consideration to achieve balance link load. Let $\alpha$ and $\beta$ be the thresholds of R3 interface and R4 interface loads, and $\mathrm{x}$ and $\mathrm{y}$ be the current loads measured by the serving ASN GW in R3 and R4, respectively. We denote the link capacities of R3 and R4 by $C_{R 3}$ and $C_{R 4}$, respectively. Then the proposed heuristic decision approach is as follows:

$$
\begin{array}{cl}
\text { i. } & \text { if }(x \geq \alpha) \cap(y<\beta) \rightarrow \text { ASN anchored mobility } \\
\text { ii. } & \text { if }(x<\alpha) \cap(y \geq \beta) \rightarrow \text { SIP mobility } \\
\text { iii. } & \text { if }(x<\alpha) \cap(y<\beta) \rightarrow \text { ASN anchored mobility (if }(x / \alpha)>(y / \beta)) \text {; SIP mobility } \\
& \text { (otherwise) } \\
\text { iv. } & \text { if }(x \geq \alpha) \cap(y \geq \beta) \rightarrow \text { ASN anchored mobility (if }\left(x / C_{R 3}\right)>\left(y / C_{R 4}\right) \text {; SIP mobility } \\
& \text { (otherwise) }
\end{array}
$$

It is noted that the handoff connection will be dropped if there is no available bandwidth in R3 and R4 interfaces.

\section{EXPERIMENTS AND SimULATION RESULTS}

In order to evaluate the performance of the proposed scheme, exhaustive simulations were carried out. Our simulation topology is shown in Figure 8. The environment includes seven ASNs and every ASN has one ASN-GW and one BS. All ASN will connect to the Internet through the egress router.

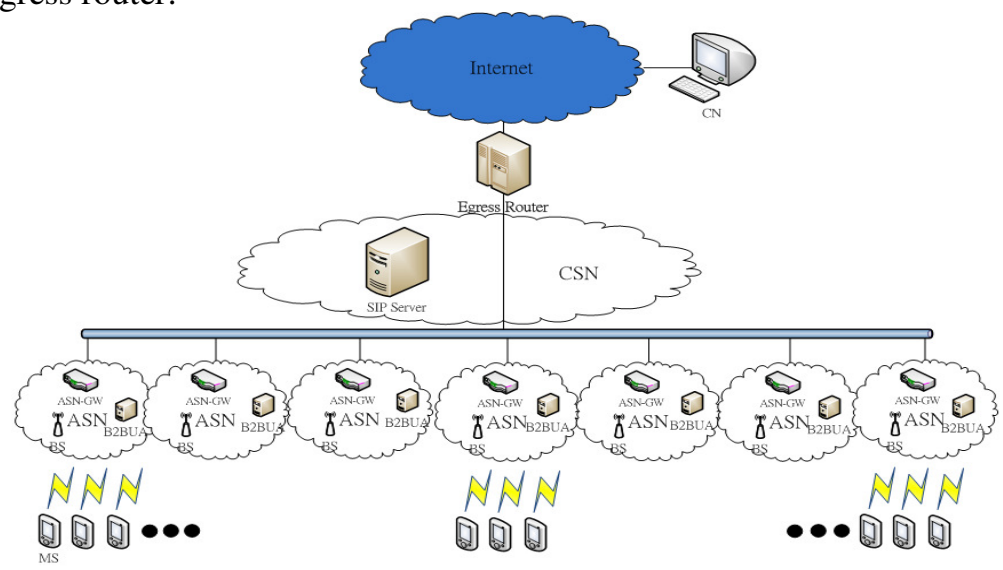

Fig. 8. The simulation architecture 
The ASN profile B, which means the interfaces between ASN-GW and BS are a black box, was adopted in our simulations. We focus on the core network loading balance problem and the R6 interface between the ASN-GW and BS is not considered in our simulations. We assume that each ASN-GW and ASN GW pair has separated R4 bandwidth. And the bandwidth of each R3 interface from each ASN-GW to the egress router is independent of each other. Figure 9 illustrates the arrangement of the above seven ASN-GWs viewed from top. Every BS may adopt one of the four modulations, which are 64QAM, 16QAM, QPSK and BPSK, according to channel condition between MS and BS. In the neighbourhood BS, every modulation has some overlaps. The MS may initiate handoff when it travels in this overlapped area. The channel condition is assumed to be proportional to the distance between MS and BS and the modulation and coding scheme is dynamically adapted according to the channel condition, as shown in Table 1. The MS is assumed to move according to the random way point mobility model in our simulations. The frame duration is $5 \mathrm{~ms}$. The mobility related parameters are listed in Table 2. The simulation results of the proposed hybrid ASN+SIP scheme is compared with the drastic algorithm, which ignores the link loads and adopts either the ASN anchored mobility (ASN only) or SIP mobility (SIP only), to illustrate the performance of our approach.

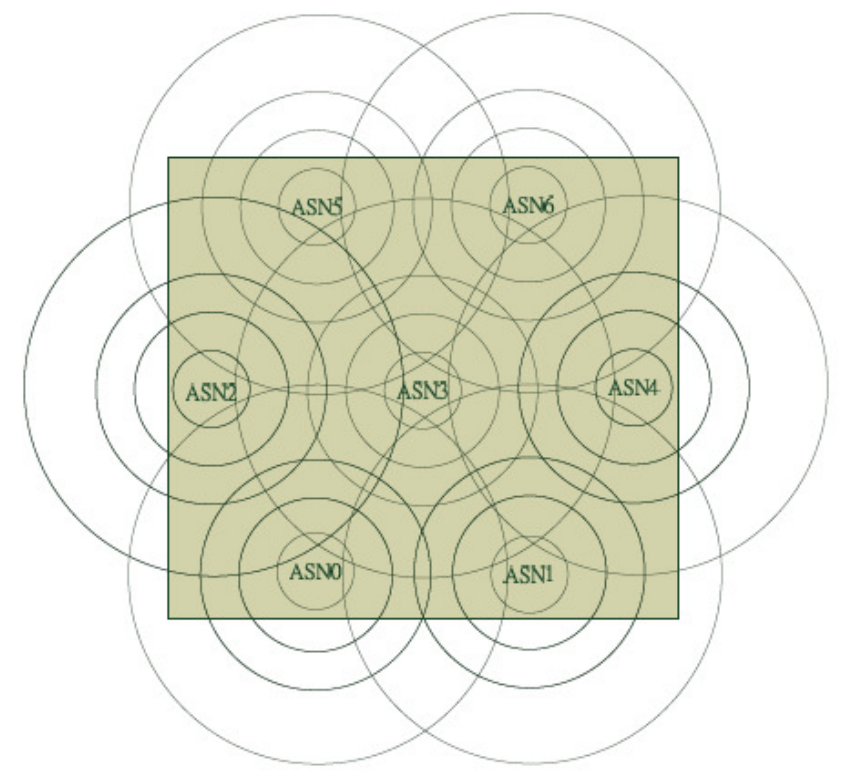

Fig. 9. Top view of the simulation 1 and 2 environment

Table 1. Modulation and BS coverage radius

\begin{tabular}{|l|l|l|l|l|}
\hline Coverage Radius & $\begin{array}{l}\text { Inner } \\
\text { circle }\end{array}$ & $\begin{array}{l}\text { Second } \\
\text { circle }\end{array}$ & $\begin{array}{l}\text { Third } \\
\text { circle }\end{array}$ & Forth circle \\
\hline Modulation/Coding & $64 \mathrm{QAM}$ & 16QAM & QPSK & BPSK \\
\hline Distance & $1 \mathrm{Km}$ & $2 \mathrm{Km}$ & $3 \mathrm{Km}$ & $5 \mathrm{Km}$ \\
\hline
\end{tabular}


Table 2. Parameters of mobility model

\begin{tabular}{|l|l|}
\hline Position & Uniform Distribution \\
\hline Speed & $\begin{array}{l}0 \sim 120 \mathrm{Km} / \text { Hr Uniform } \\
\text { Distribution }\end{array}$ \\
\hline Direction & $\begin{array}{l}0 \sim 360 \text { degree Uniform } \\
\text { Distribution }\end{array}$ \\
\hline Halt time & $\begin{array}{l}\text { Exponential Distribution } \\
\text { Mean value }: 15 \text { second } \\
\text { Upper Bound }: 30 \text { second } \\
\text { Lower Bound : 3 second }\end{array}$ \\
\hline Max Retry & 3 \\
\hline Retry Interval & $200 \mathrm{~ms}$ \\
\hline Model & Random Waypoint Mobility \\
\hline Threshold & $80 \%$ \\
\hline
\end{tabular}

As mentioned in the previous section, a handoff connection will be dropped if there is no available R3 or R4 bandwidth. However, the handoff connection will also be dropped if the bandwidth of R1 air interface is saturated. Therefore, we conducted two experiments to verify the performance of the proposed scheme. In experiment 1, the bandwidth of R1 interface is assumed to be unlimited. This arrangement is to prevent the limited R1 capacity from affecting the dropping rate introduced by the insufficient R3 and R4 links. In experiment 2, the bandwidth of R1 interface is constrained, which is more comparable to the practical environment. The number of mobile stations is 100 in both experiments.

\section{- Experiment 1:}

The requested bandwidth of each MS is Gaussian distributed with mean loads varying from 1 to $13 \mathrm{Mbps}$, where the standard deviation is one. The bandwidth capacities of R3 and R4 are 200 Mbps and $15 \mathrm{Mbps}$, respectively. The values of $\alpha$ and $\beta$ are set to be $80 \%$ of the link capacity. As one ASN-GW connects to six ASN-GWs, the R4 of ASN-GW total bandwidth of ASN 3 is 90 Mbps. The simulation is illustrated in the Figure 10.

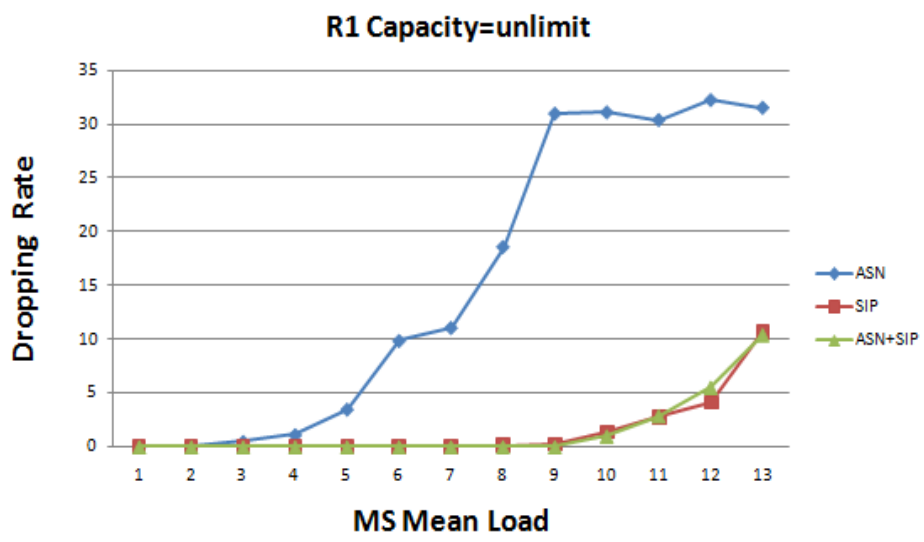

Fig. 10. Experiment 1 simulation results (unlimited R1 bandwidth) 
International Journal of Computer Networks \& Communications (IJCNC) Vol.3, No.5, Sep 2011

Figure 10 indicates that the dropping rate of the ASN anchored mobility only scheme is the highest. It is mainly due to the limited bandwidth capacity in R4 interface. The SIP mobility only and the proposed ASN+SIP scheme have similar dropping rate. Because the capacity of R3 interface is much larger than the R4 interface and the SIP mobility scheme always choose SIP mobility, therefore the dropping rate decreases. Furthermore, since the proposed scheme can dynamically choose R3 or R4 interface, its dropping rate is also lower accordingly.

\section{- Experiment 2:}

In this experiment, we constrain the R1 interface capacity to be $100 \mathrm{Mbps}$ and the mean bandwidth of each MS requested varies from 1 to $6 \mathrm{Mbps}$. The main reason of reducing the mean load of each MS is the limited bandwidth in air interface. The other simulation parameters are the same as in experiment 1 . The purpose of this experiment is to investigate whether the limitation on the R1 capacity affects the performance of those schemes or not. The simulation results of the three schemes are illustrated in the Figure 11.

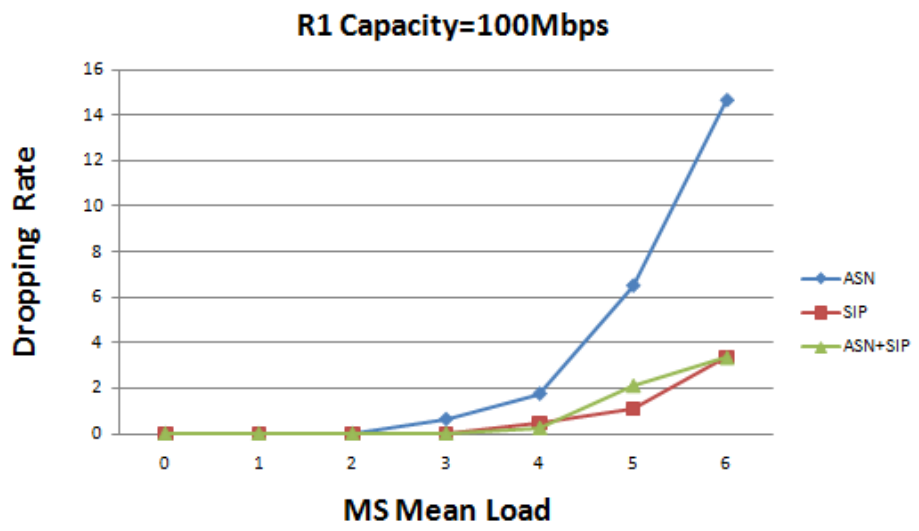

Fig. 11. Experiment 2 simulation results (R1 bandwidth=100 Mbps)

Figure 11 shows that the dropping rate of the ASN only scheme is still much higher than the SIP only and the proposed ASN+SIP schemes. Compared with Figure 9 for the mean load of 6 Mbps, the dropping rates of the SIP only and ASN+SIP schemes approach 0 in experiment $1 \mathrm{~m}$ and it is about $3 \%$ in experiment 2 . However, the dropping rate of ASN only scheme increases from $10 \%$ to $15 \%$. It indicates that although the R1 capacity affects the dropping rate, the dropping rate will increase dramatically if the selection of R3 and R4 interfaces for the handoff connection is not properly considered.

It should be pointed out that although the SIP only and the proposed ASN+SIP schemes have similar dropping rates for both experiments, it is clear that the handoff delay and packet loss rate of the proposed scheme would be lower than the SIP only scheme. This is because the ASN always needs to initiate re-invite procedure during handoff.

In the follow experiments we limit the MS moving area to avoid MS move non-coverage area and set BS using BPSK with coverage of 3.15KM as shown in Figure 12 and Table 3. 


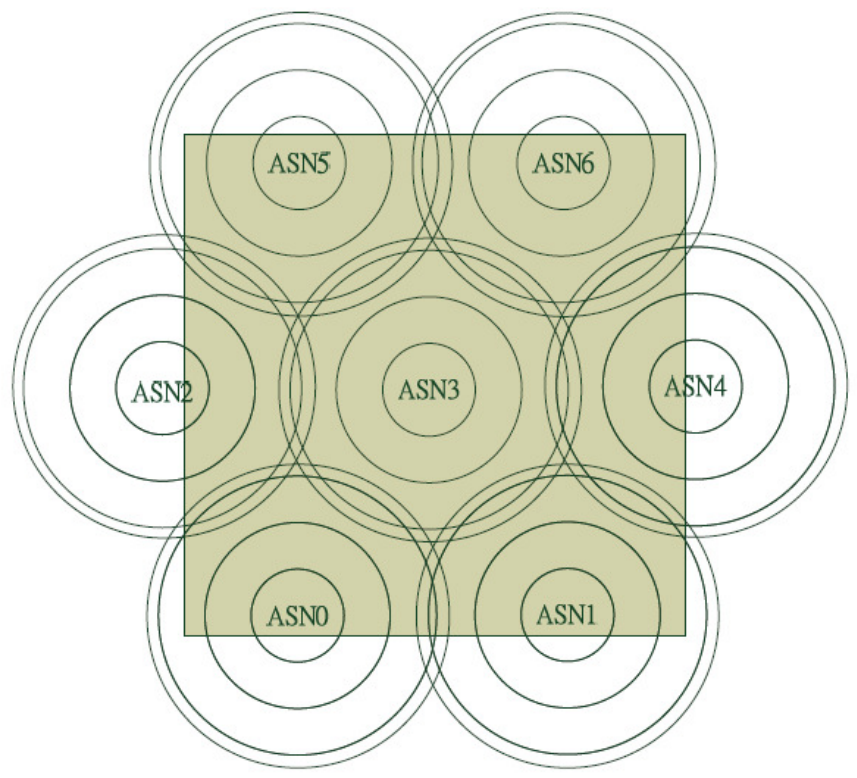

Fig. 12. Top view of the simulation 3 and 4 environment

Table 3. Modulation and BS coverage radius

\begin{tabular}{|l|l|l|l|l|}
\hline Coverage Radius & $\begin{array}{l}\text { Inner } \\
\text { circle }\end{array}$ & $\begin{array}{l}\text { Second } \\
\text { circle }\end{array}$ & $\begin{array}{l}\text { Third } \\
\text { circle }\end{array}$ & Forth circle \\
\hline Modulation/Coding & 64QAM & 16QAM & QPSK & BPSK \\
\hline Distance & $1 \mathrm{Km}$ & $2 \mathrm{Km}$ & $3 \mathrm{Km}$ & $3.15 \mathrm{Km}$ \\
\hline
\end{tabular}

\section{- Experiment 3:}

In order to compare the BS coverage radius effect, all the parameters of the simulation are the same as experiment 1, expect for Coverage Radius of BS BPSK. The simulation result is shown in Figure 13.

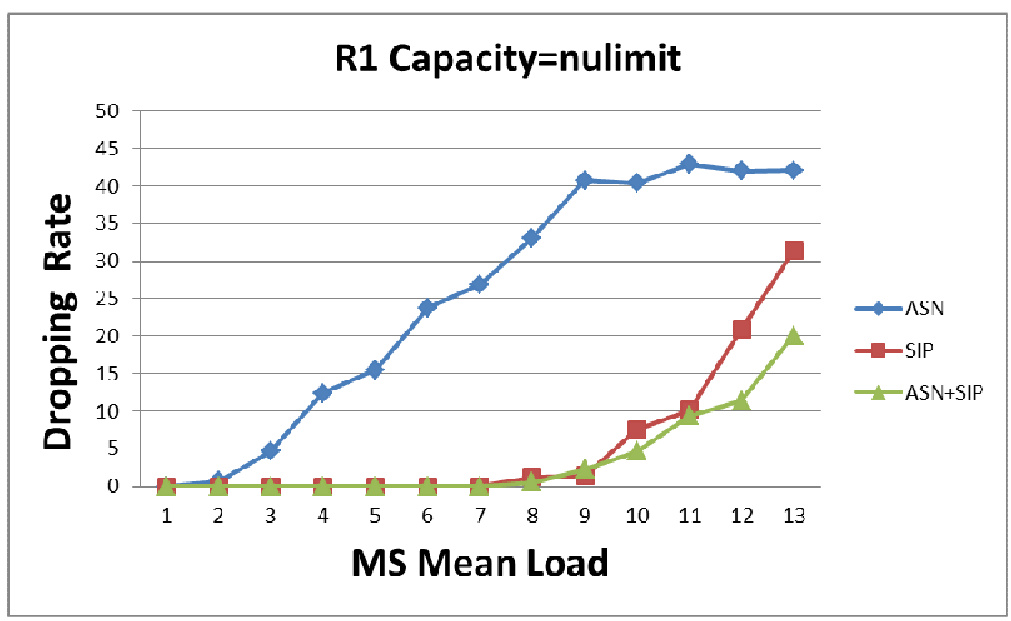

Fig. 13. Experiment 3 simulation result 
By comparing simulation results of Figure 13 and Figure 10, we observe that the dropping rate rises early in all algorithms. Since we reduce the Coverage Radius of BS BPSK, MS moves easily in the only one BS serving area. If the serving BS is overloaded, MS will be dropped. MS handoff non-serving BS area, the core network will use ASN anchored mobility. And this is the scenario in which ASN anchored mobility combining SIP mobility algorithm fully demonstrates its advantage. As network that uses ASN anchored mobility combining SIP mobility algorithm can serve more MSs, when R3 loading is more overloaded than R4's. Hence Figure 13 shows that when mean load of MS is 13 Mbps, using SIP mobility dropping rate is higher than using ASN anchored mobility combining SIP mobility's.

\section{- Experiment 4}

As we reduce BS Coverage Radius, the occurrences of MS handoff will increase. The parameters of the simulation are the same as experiment 2 expect for Coverage Radius of BS BPSK. Then we record dropping rate from MS request bandwidth 1 to $6.5 \mathrm{Mbps}$, and average the record not including the largest and the smallest. The simulation is illustrated in the Figure 14.

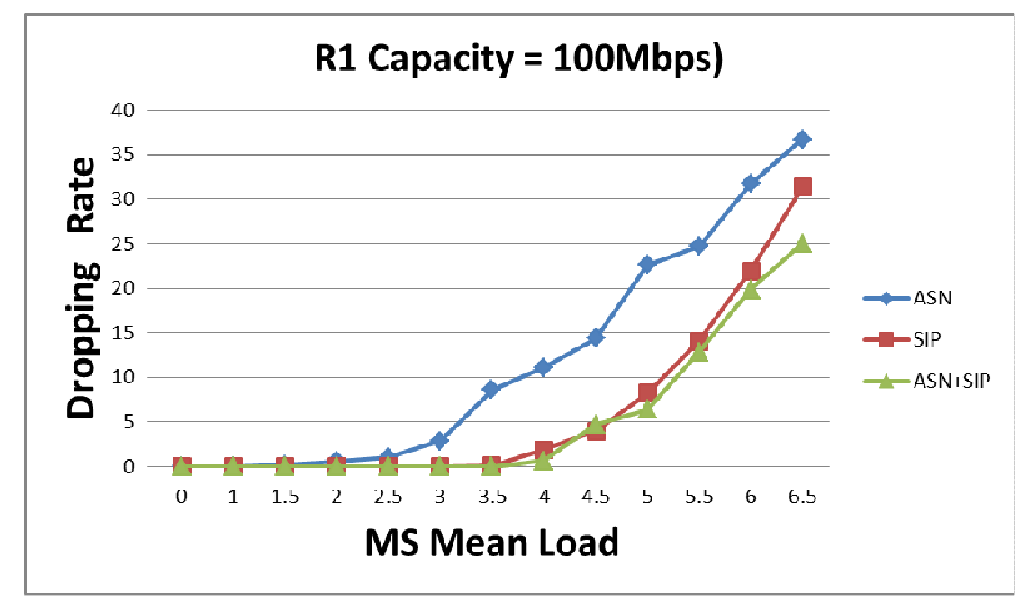

Fig. 14. Experiment 4 simulation result

We note that the results based on SIP mobility are similar to that of ASN anchored mobility combining SIP mobility algorithm. Since there is not enough R1 bandwidth capacity, the majority of MSs are dropped. Therefore balancing core network R3 and R4 loading doesn't demonstrate any discernible effect.

\section{- Experiment 5:}

In order to observe the utilization rate of core network, we increase the MS number to 700 and each bandwidth of MS is fixed to be $1.5 \mathrm{Mbps}$. Initially we allocate averagely these MSs in each ASN, so each ASN serve the 100 MSs. These MSs move randomly following Random Waypoint Model. In the related parameters, we set BS/R1 capacity to be unlimited. This is for preventing the R1 capacity from influencing the core network balance loading. In the next experiments we use the following parameters to analyze the utilization rate of R4 Link and the utilization rate of R3 Link. We set the R4 capacity to be $15 \mathrm{Mbps}$, R3 capacity to be $200 \mathrm{Mbps}$, MS load to be 1.5 Mbps and frame duration is $5 \mathrm{~ms}$. 
International Journal of Computer Networks \& Communications (IJCNC) Vol.3, No.5, Sep 2011

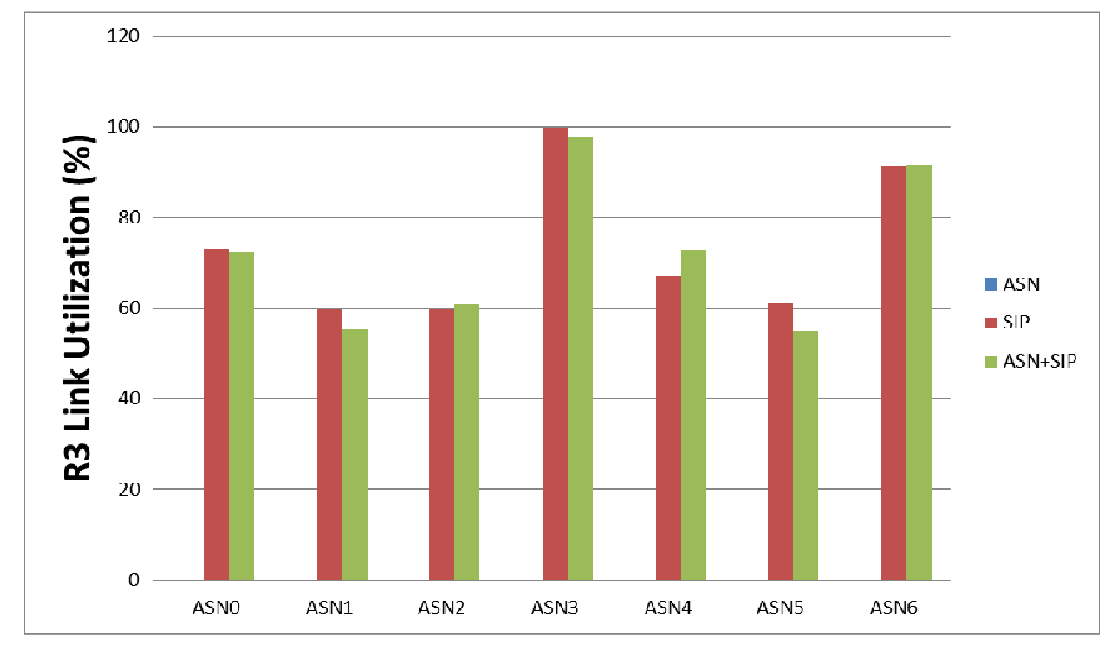

Fig. 15. R3 Link Utilization (\%)

In the Figure 15, we observe that the highest utilization rate of R3 Link happened in ASN 3. In the Figure 12 simulation topology, ASN 3 is placed in the middle of the seven ASNs, and therefore most of the MS would pass through it. In addition to the utilization rate of R3, we can see some differences between the SIP mobility and ASN anchored mobility combining SIP mobility. If we simply use the ASN R3 of SIP mobility, the utilization rate will be close to $100 \%$, This is because when the R3 of ASN 3 is overloaded, there is no way to share bandwidth through the ASN anchored mobility.

\section{Conclusions}

The paper proposes a scheme that combines the ASN anchored mobility and SIP mobility scenarios in WiMax networks as well as the feasible architecture to improve the mobility performance. There are three main contributions in this paper. It proposes SIP mobility in the WiMax architecture to achieve application layer mobility, so that MS needs not to change or update operating system kernel in order to be equipped with mobile IP capability. To achieve application layer mobility in the WiMax architecture, we design a B2BUA component to replace existing mobile IP server side architecture and reduce the transmission of packets in the air interface, thereby lowering handoff latency of the MSs and minimizing loss of control message. Finally, the proposed hybrid scheme provides the flexibility for network resource arrangement so that the network resource can be effectively allocated. The simulation results also reflect the objectives of the proposed scheme. We observe that the two thresholds, $\alpha$ and $\beta$, can be further designed to be more deliberation. Thus, the B2BUA may switch a connection with ASN anchored mobility to SIP mobility when the link utilization is too high, even when the layer 2 handoff does not yet occur. Owing to the link utilization and load balancing issues, more heuristic handoff considerations will be feasible and this will be our on-going works.

\section{ACKNOWLEDGEMENTS}

This research work was supported in part by the grants from the Ministry of Education and National Science Council (NSC) (grant numbers: NSC 97-2221-E-008-033, NSC 98-2221-E008-063, and NSC 99-2221-E-008-005). 
International Journal of Computer Networks \& Communications (IJCNC) Vol.3, No.5, Sep 2011

\section{REFERENCES}

[1] IEEE Standard for Local and metropolitan area networks Part 16: Air Interface for Fixed and Mobile Broadband Wireless Access Systems Amendment 2: Physical and Medium Access Control Layers for Combined Fixed and Mobile Operation in Licensed Bands and Corrigendum 1, IEEE Std 802.16e-2005 and IEEE Std 802.16-2004 / Cor 1-2005 (2006)

[2] Draft Standard for Local and metropolitan area networks Part 16: Air Interface for Broadband Wireless Access Systems, P802.16Rev2/D2 (2007)

[3] Ray, S.K.,Pawlikowski, K.,Sirisena, H.; "Handoff in Mobile WiMAX Networks: The State of Art and Research Issues", in IEEE Communications Surveys \& Tutorials

[4] Seyyedoshohadaei, S.M. ; Khatun, S. ; Ali, B.M. ; Othman, M. ; Anwar, F. ; “An integrated scheme to improve performance of fast mobile IPv6 handover in IEEE 802.16e network", in Communications (MICC), IEEE 9th Malaysia International Conference (2009)

[5] M. Handley, H. Schulzrinne, E. Schooler, and J. Rosenberg, "SIP: session initiation protocol," Request for Comments (Proposed Standard) 2543, Internet Engineering Task Force, March (1999)

[6] E. Wedlund and H. Schulzrinne, "Mobility Support Using SIP” , Proc. 2nd ACM/IEEE Int'1. Wksp. Wireless and Mobile Multimedia, August (1999)

[7] N.Nakajima, A. Dutta, S. Das, H. Schulzrinne, "Handoff delay analysis and measurement for SIP based mobility in IPv6”, Communications, 2003. ICC '03, May (2003)

[8] Yen-Wen Chen and Ming-Jen Huang, "Study of Heuristic MAP Selection and Abstraction Schemes with Load Balance in HMIPv6," Wireless Personal Communications, on-line version, DOI: $10.1007 / \mathrm{s} 11277-009-9854-5$ (2010)

[9] Fu-Yia Hsieh, Yeh-Wen Chen, and Po-Wei Wu, "Cross Layer Design of Handoffs in IEEE 802.16e Network", 2006 International Computer Symposium conference,29 Jan. (2007)

[10] Shaoh-Chen Ke, Yen-Wen Chen, and Shih-Yu Hsu, “A Performance Comparison of Inter-ASN Handover Management Schemes over Mobile WiMAX Network," International Journal of Wireless Communications and Networking (IJWCN), pp. 75-85, June (2009)

[11] WiMAX Network Architecture -Stage 2-Release 1.1.0, WiMAX Forum, July (2007)

[12] WiMAX Network Architecture -Stage 3-Release 1.1.0, WiMAX Forum, July (2007)

\section{Authors}

Yen-Wen Chen received the Ph.D. degree in Electronic Engineering from National Taiwan University of Science and Technology (NTUST) in 1997. During 1983 to 1998, he worked at Chunghwa Telecommunication Laboratories, Taiwan and was a project manager of the broadband switching systems. From August 1998 to July 2000, he joined the Department of Information Management, Central Police University. Since August 2000, Dr. Chen has joined the Department of Communication Engineering of National Central University. Currently, he is an associate professor. His research interests include broadband mobile networks, QoS management, and GMPLS. Dr. Chen is a member of the IEEE communication society.

Meng-Hsien Lin was born in 1976. He received the M.S. degree in electrical engineering from National Chung Cheng University, Taiwan, in 2000. He is working toward the Ph.D. degree in communication engineering at the National Central University, Taiwan. His research interests include Computer Networks, Network Security, and Cloud Computing. He is currently with Chunghwa Telecommunication Laboratories, Taiwan.

Hong-Jang Gu was born in 1984. He received the M.S. degree in communication engineering from National Central University, Taiwan, in 2008. His research interests include Computer Networks, Network Security. He is currently with Gemtek Corporation, Taiwan. 